University of Nebraska - Lincoln

DigitalCommons@University of Nebraska - Lincoln

3-9-2006

\title{
Magnetic ordering in Gd monopnictides: Indirect exchange versus superexchange interaction
}

\author{
Chun-gang Duan \\ University of Nebraska-Lincoln, cgduan@clpm.ecnu.edu.cn \\ Renat F. Sabiryanov \\ University of Nebraska - Omaha, rsabirianov@mail.unomaha.edu \\ Wai-Ning Mei \\ University of Nebraska at Omaha, physmei@unomaha.edu \\ Peter A. Dowben \\ University of Nebraska-Lincoln, pdowben@unl.edu \\ Sitaram Jaswal \\ University of Nebraska, sjaswal1@unl.edu \\ See next page for additional authors
}

Follow this and additional works at: https://digitalcommons.unl.edu/physicsdowben

Part of the Physics Commons

Duan, Chun-gang; Sabiryanov, Renat F.; Mei, Wai-Ning; Dowben, Peter A.; Jaswal, Sitaram; and Tsymbal, Evgeny Y., "Magnetic ordering in Gd monopnictides: Indirect exchange versus superexchange interaction" (2006). Peter Dowben Publications. 2.

https://digitalcommons.unl.edu/physicsdowben/2

This Article is brought to you for free and open access by the Research Papers in Physics and Astronomy at DigitalCommons@University of Nebraska - Lincoln. It has been accepted for inclusion in Peter Dowben Publications by an authorized administrator of DigitalCommons@University of Nebraska - Lincoln. 


\section{Authors}

Chun-gang Duan, Renat F. Sabiryanov, Wai-Ning Mei, Peter A. Dowben, Sitaram Jaswal, and Evgeny Y. Tsymbal 


\title{
Magnetic ordering in Gd monopnictides: Indirect exchange versus superexchange interaction
}

\author{
Chun-Gang Duan ${ }^{\mathrm{a}}$ \\ Department of Physics, University of Nebraska-Lincoln, Lincoln, Nebraska 68588 \\ and Center for Materials Research Analysis, University of Nebraska-Lincoln, Lincoln, Nebraska 68588 \\ R. F. Sabiryanov and W. N. Mei \\ Department of Physics, University of Nebraska at Omaha, Omaha, Nebraska 68182 \\ P. A. Dowben, S. S. Jaswal, and E. Y. Tsymbal \\ Department of Physics, University of Nebraska-Lincoln, Lincoln, Nebraska 68588 \\ and Center for Materials Research and Analysis, University of Nebraska-Lincoln, Lincoln, Nebraska 68588
}

(Received 9 March 2006; accepted 17 March 2006; published online 4 May 2006)

\begin{abstract}
The exchange interaction parameters of $\mathrm{Gd}$ monopnictides are deduced from fitting the total energies of different magnetic configurations to those computed within the Heisenberg model. The magnetic structures predicted by first-principles calculations as well as the Curie (Néel) temperatures obtained from Monte Carlo simulations are both in good agreement with experiments. A detailed analysis of the exchange parameters suggests that the Ruderman-Kittel-Kasuya-Yosida-type indirect exchange interactions and antiferromagnetic superexchange interactions coexist in these compounds. The magnetic order changes from ferromagnetic in $\mathrm{GdN}$ to antiferromagnetic in other $\mathrm{Gd}$ pnictides as a result of the increased ionic radius of a pnictide in the latter. (C) 2006 American Institute of Physics. [DOI: 10.1063/1.2200767]
\end{abstract}

Magnetic ordering of the rare-earth pnictides $R X(R$ is a rare-earth element and $X=\mathrm{N}, \mathrm{P}, \mathrm{As}, \mathrm{Sb}, \mathrm{Bi}$ ) has long been of interest because of their "coupled" magnetic and electronic properties. ${ }^{1-14}$ This interest is enhanced by the wide industrial applications of the rare-earth pnictides ${ }^{15}$ with possible use as spin filters and robust magnetoresistive materials for spintronics. ${ }^{16}$

Even after more than 40 years efforts, ${ }^{1-14}$ a thorough understanding of the magnetic ordering of $R X$ compounds, with simple NaCl-type structure, is still elusive. The persistent difficulty is finding an accurate description of the highly localized open shell $4 f$ electrons, which is a key to understanding the magnetic properties and other fascinating many-body characteristic of the rare earths and rare-earth compounds. The $4 f$ electrons are well localized and the direct $4 f-4 f$ exchange is negligible. Thus, in the rare-earth metals, the coupling between neighboring magnetic ions occurs through an indirect magnetic exchange of the Ruderman-Kittel-KasuyaYosida (RKKY) type. ${ }^{17}$ The situation in the $R X$ system is much more complicated. The presence of the pnictide greatly decreases the free charge carrier density and introduces more complicated exchange mechanisms such as superexchange interactions. ${ }^{18}$ In addition, in the face-centered cubic (fcc) structure, the two nearest neighboring (NN) atoms of a given atom can themselves be NN atoms, which often causes spin frustration. The combination of all the factors that contribute to the magnetic ordering in rare-earth pnictides renders the problem extremely complex. This complexity is, however, reduced in gadolinium pnictides, where the $\mathrm{Gd} f$ orbitals are half occupied, hence the orbital angular momentum is zero. Consequently, the anisotropic and multipole effects are small, thus making the analysis of the magnetic exchange interactions in $\mathrm{Gd} X$ compounds less demanding.

In a recent letter, ${ }^{19}$ we have predicted that the electronic and magnetic properties of $\mathrm{GdN}$ are extremely sensitive to

\footnotetext{
${ }^{a)}$ Electronic mail: wxbdcg@gmail.com
}

the strain effect, resulting in a transformation of this compound from half-metallic to semimetallic and then ultimately to semiconducting with the lattice expansion. In this letter, using first-principles total energy calculations, we present a systematic theoretical study of the magnetic exchange interactions on the whole $\mathrm{Gd}$ monopnictide family, $\mathrm{Gd} X(X$ $=\mathrm{N}, \mathrm{P}, \mathrm{As}, \mathrm{Sb}, \mathrm{Bi})$. Following description of Ref. 20, we consider four different magnetic ordering configurations: ferromagnetic (FM) and three antiferromagnetic (AFM). The latter are shown in Fig. 1 and denoted by AFM-I, AFM-II, and AFM-III. We predict that the $\mathrm{Gd} X$ magnetic structure changes from FM in GdN to AFM-II in other GdX compounds in agreement with experimental observations. We demonstrate that this behavior is caused by the coexistence of the RKKY-type indirect exchange interactions and superexchange interactions, the relative contribution of which changes with the ionic radius of pnictides.

Our first-principles calculations are based on density functional theory implemented within the full-potential linear-augmented-plane-wave method. ${ }^{21}$ We use a local spin density approximation (LSDA) with additional Hubbard correlation terms $U$ and $J$ associated with the $4 f$ narrow bands (the so called LSDA $+U$ approach). ${ }^{22}$ Following the previous work, ${ }^{19}$ we assume that $U=6.7 \mathrm{eV}$ and $J=0.7 \mathrm{eV}$. Since the energy difference between different magnetic ordering states is small (of the order of a few meV), we use $4000 k$ points in

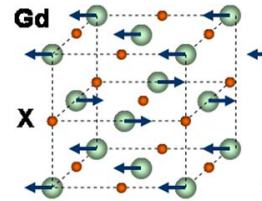

(a)

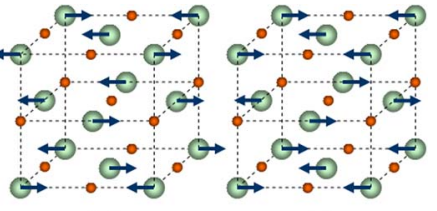

(b) (c)
FIG. 1. (Color online) Three antiferromagnetic ordering configurations in Gd pnictides: (a) AFM-I, (b) AFM-II, and (c) AFM-III. The arrows indicate magnetic moment orientations on Gd atoms. 
TABLE I. Calculated total energy differences per formula between ferromagnetic and three antiferromagnetic configurations for Gd monopnictides $\Delta E_{n}$ $=E_{n}-E_{\mathrm{FM}}(n=\mathrm{I}, \mathrm{II}, \mathrm{III})$. The exchange parameters, $J_{1}, J_{2}$, and $J_{3}$ are obtained according to Eq. (2). Values inside the parentheses are calculated using the theoretical lattice constants. Theoretical and experimental transition temperatures (Ref. 14) are listed for comparison.

\begin{tabular}{|c|c|c|c|c|c|c|c|c|c|}
\hline & \multirow[b]{2}{*}{$\begin{array}{c}a(\AA) \\
\text { Expt. (Theor.) }\end{array}$} & \multirow[b]{2}{*}{$\begin{array}{c}\Delta E_{\mathrm{I}} \\
(\mathrm{meV})\end{array}$} & \multirow[b]{2}{*}{$\begin{array}{c}\Delta E_{\mathrm{II}} \\
(\mathrm{meV})\end{array}$} & \multirow[b]{2}{*}{$\begin{array}{l}\Delta E_{\mathrm{III}} \\
(\mathrm{meV})\end{array}$} & \multirow[b]{2}{*}{$\begin{array}{c}J_{1} \\
(\mathrm{meV})\end{array}$} & \multirow[b]{2}{*}{$\begin{array}{c}J_{2} \\
(\mathrm{meV})\end{array}$} & \multirow[b]{2}{*}{$\begin{array}{c}J_{3} \\
(\mathrm{meV})\end{array}$} & \multicolumn{2}{|c|}{$T_{N(C)}(\mathrm{K})$} \\
\hline & & & & & & & & $\mathrm{MC}$ & Expt. \\
\hline GdN & 4.97(4.92) & $6.7(7.1)$ & $4.2(5.1)$ & $6.5(7.3)$ & $0.86(0.95)$ & $-0.14(-0.04)$ & $-0.01(-0.03)$ & $34(38)$ & 58.0 \\
\hline $\mathrm{GdP}$ & $5.71(5.63)$ & $-2.8(-3.3)$ & $-6.5(-6.9)$ & $-3.5(-4.0)$ & $-0.17(-0.21)$ & $-0.74(-0.74)$ & $-0.09(-0.11)$ & $12(13)$ & 15.9 \\
\hline GdAs & $5.86(5.77)$ & $-3.9(-4.3)$ & $-8.4(-8.8)$ & $-4.7(-5.4)$ & $-0.22(-0.34)$ & $-0.91(-0.93)$ & $-0.13(-0.10)$ & $14(16)$ & 18.7 \\
\hline $\mathrm{GdSb}$ & $6.22(6.11)$ & $-5.7(-6.6)$ & $-11.1(-11.5)$ & $-7.2(-7.9)$ & $-0.51(-0.60)$ & $-1.13(-1.10)$ & $-0.10(-0.11)$ & $20(19)$ & 23.4 \\
\hline $\mathrm{GdBi}$ & $6.30(6.24)$ & $-6.9(-7.3)$ & $-13.4(-13.8)$ & $-8.8(-9.3)$ & $-0.66(-0.71)$ & $-1.37(-1.38)$ & $-0.10(-0.10)$ & $22(23)$ & 25.8 \\
\hline
\end{tabular}

the Brillouin zone to obtain the total energy accuracy of $0.1 \mathrm{meV}$.

Our basic strategy is to first deduce the exchange interaction parameters of $\mathrm{Gd} X$ compounds by fitting the firstprinciples total energies of different magnetic configurations to those computed within the Heisenberg model,

$$
H=-\sum_{n=1,2,3} J_{n} \sum_{i>j}^{n \text {th NN }} \mathbf{S}_{i} \cdot \mathbf{S}_{j} .
$$

Here $\mathbf{S}_{i}$ is the unit vector in the direction of the magnetic moment at the $i$ th lattice site, $J_{n}$ is the exchange parameter between the $n$th NN magnetic atoms, and we limit our consideration to third NN interactions. In this case the difference between the energy of the three AFM states $E_{n}(n=\mathrm{I}, \mathrm{II}, \mathrm{III})$ shown in Fig. 1 and the energy of the FM state $E_{\mathrm{FM}}$ are

$$
\begin{aligned}
& \Delta E_{\mathrm{I}} \equiv E_{\mathrm{I}}-E_{\mathrm{FM}}=8 J_{1}+16 J_{3}, \\
& \Delta E_{\mathrm{II}} \equiv E_{\mathrm{II}}-E_{\mathrm{FM}}=6 J_{1}+6 J_{2}+12 J_{3}, \\
& \Delta E_{\mathrm{III}} \equiv E_{\mathrm{III}}-E_{\mathrm{FM}}=8 J_{1}+2 J_{2}+8 J_{3} .
\end{aligned}
$$

The calculated total energy differences $\Delta E_{n}$ and the resulting exchange parameters, for both experimental and theoretical lattice constants, are listed in Table I. The most important result, evident from Table I, is that our calculations predict correctly the switching from FM ordering of $\mathrm{GdN}$ to AFM ordering in the other gadolinium pnictides, GdP, GdAs, $\mathrm{GdSb}$, and GdBi. Moreover, we find that in all these compounds, the total energies of three AFM configurations have a consistent order, i.e., $E_{\mathrm{II}}<E_{\mathrm{III}}<E_{\mathrm{I}}$. This agrees with the experimental observations that all the AFM compounds studied here have the AFM-II structure. ${ }^{14}$ Table I also shows that, except $J_{1}$ for $\mathrm{GdN}$, all the exchange parameters have negative values, indicating that the exchange interactions in these compounds are antiferromagnetic. With the increase of anion size, as $X$ changes from $\mathrm{N}$ to $\mathrm{Bi}$, both $J_{1}$ and $J_{2}$ decrease rapidly, while the absolute change of $J_{3}$ is quite small.

To understand the nature of the exchange interactions in the Gd pnictides, we calculated the dependence of the exchange parameters on the lattice strain. As is evident from Fig. 2, for all the $\operatorname{Gd} X$ compounds, the absolute value of $J_{1}$ increases with the decrease of the lattice constant. This behavior is the signature of the RKKY-type indirect exchange interaction. Indeed, the RKKY-type coupling varies sensitively with the density of charge carriers. The enhancement of the exchange coupling between first NN magnetic sites, when the lattice constant decreases, is caused by the increased number of free charge carriers as the $\operatorname{Gd} X$ compound becomes more metallic. On the contrary, the $J_{2}$ is weakly dependent on the lattice strain for all the Gd pnictides, except GdN where it decreases appreciably with the increase of the lattice constant. As was explained in Ref. 19, this behavior signifies the strengthening of the AFM superexchange interaction when the system becomes less metallic.

To obtain a more insight into the strength of the superexchange interaction contributing to $J_{2}$ we use a model for charge-transfer-type superexchange developed for transitionmetal oxides in Ref. 23. Since the induced $d$ moment on Gd $\left(n_{d}\right)$ due to atomic $4 f-5 d$ exchange interactions is a small fraction of $\mu_{B}$ compared with an integral value in transitionmetal oxides, we scale the superexchange formula in Ref. 23 with $n_{d}$. The value of $n_{d}$ is estimated by subtracting the ideal $\mathrm{Gd}^{3+}$ spin moment, i.e., 7, from the calculated total spin moment on Gd ions. Thus an expression for the superexchange interaction takes the form

$$
J_{2}^{\text {super }}=-n_{d} \frac{t_{p d}^{4}}{\Delta^{3}},
$$

where $\Delta$ is the energy difference between the Gd $5 d$ orbital and the outmost $p$ state of $X$ ion and the hopping parameter $t_{p d}$ can be evaluated according to Harrison. ${ }^{24}$ Table II shows the so calculated $J_{2}^{\text {super }}$ of the $\operatorname{Gd} X$ compounds at their experimental lattice constants, together with the corresponding values of $t_{p d}, \Delta, n_{d}, \mathrm{Gd}$ ion magnetic moment $\mu_{\mathrm{Gd}}$, anion size $R_{X}$, and interatomic distance $R_{i j}$ used in the calculation. We see from Table II that our qualitative estimate of $J_{2}^{\text {super }}$ is of the order of $J_{2}$ for all the $\mathrm{GdX}$ compounds. Thus, the second $\mathrm{NN}$ exchange parameters seem to be primarily due to the superexchange interaction. GdN may be the only exclusion from this rule-the decrease of $J_{2}$ versus lattice strain indicates the weakening of the indirect exchange coupling. ${ }^{19}$
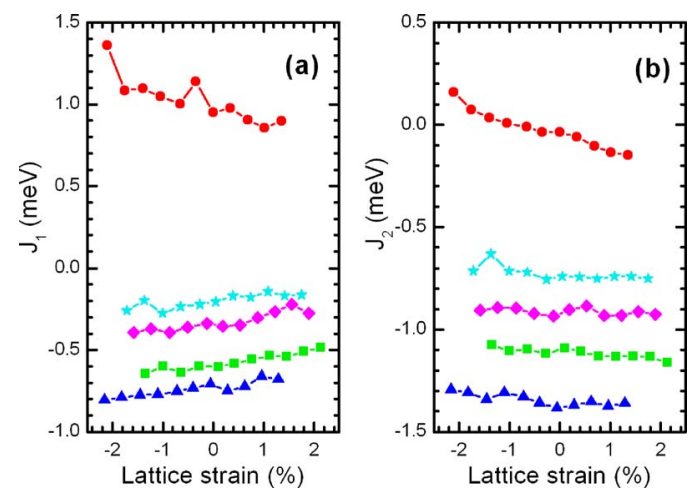

FIG. 2. (Color online) Calculated exchange parameters $J_{1}$ (a) and $J_{2}$ (b) for Gd pnictides as a function of the lattice strain: GdN (circles), GdP (stars), GdAs (diamonds), GdSb (squares), and GdBi (triangles). The lattice strain is defined by the relative deviation of the lattice constant from the theoretical equilibrium lattice constant. 
TABLE II. Estimated superexchange energies $J_{2}^{\text {super }}$ between the second nearest neighboring $\mathrm{Gd}$ ions on the experimental structures of $\mathrm{Gd} X$ and parameters used for this estimate: the anion size $R_{X}$, the interatomic distance $R_{i j}$, Gd ion magnetic moment $\mu_{\mathrm{Gd}}$, the occupation number $n_{d}$ of the $d$ orbital, the hopping parameter $t_{p d}$, and the energy difference $\Delta$ between the Gd $5 d$ orbital, and the outmost $p$ state of $X$ ion. The radius of $\mathrm{Gd} 5 d$ orbital is $1.78 \AA$.

\begin{tabular}{cccccccc}
\hline \hline & $\begin{array}{c}R_{X} \\
(\AA)\end{array}$ & $\begin{array}{c}R_{i j} \\
(\AA)\end{array}$ & $\begin{array}{c}\mu_{\mathrm{Gd}} \\
\left(\mu_{B}\right)\end{array}$ & $n_{d}$ & $\begin{array}{c}t_{p d} \\
(\mathrm{eV})\end{array}$ & $\begin{array}{c}\Delta \\
(\mathrm{eV})\end{array}$ & $\begin{array}{c}J_{2}^{\text {super }} \\
(\mathrm{meV})\end{array}$ \\
\hline $\mathrm{GdN}$ & 0.70 & 2.48 & 7.038 & 0.038 & 0.754 & 3.4 & -0.31 \\
$\mathrm{GdP}$ & 1.07 & 2.85 & 7.068 & 0.068 & 0.637 & 2.5 & -0.72 \\
$\mathrm{GdAs}$ & 1.15 & 2.93 & 7.076 & 0.076 & 0.611 & 2.4 & -0.77 \\
$\mathrm{GdSb}$ & 1.33 & 3.11 & 7.096 & 0.096 & 0.552 & 2.1 & -0.96 \\
$\mathrm{GdBi}$ & 1.37 & 3.15 & 7.104 & 0.104 & 0.540 & 2.0 & -1.11 \\
\hline \hline
\end{tabular}

Using Monte Carlo (MC) simulations based on the Heisenberg Hamiltonian (1) and the first-principles exchange parameters we calculated the transition temperatures of the $\mathrm{Gd} X$ compounds (the Curie temperature $T_{C}$ for $\mathrm{GdN}$ and the Néel temperatures $T_{N}$ for the other Gd pnictides). In the MC simulations we used a $10 a \times 10 a \times 10 a$ supercell (which includes 4000 spins) with periodic boundary conditions (here $a$ is the lattice constant). As is evident from Table I, our MC simulations not only accurately reproduce the experimentally observed ground states for these five compounds but also predict the transition temperatures in good agreement with experiments. Given the strong correlated nature of the $\mathrm{Gd}$ pnictides, this agreement is quite impressive, supporting the validity of our first-principles results.

Following the above analysis, a whole picture on the magnetic coupling in $\operatorname{Gd} X$ systems can be described as follows. As we know, Gd metal with hep structure is ferromagnetic at room temperature $\left(T_{C}=293.2 \mathrm{~K}\right) .^{25}$ The NN distance of bulk Gd at the experimental lattice constant is $3.63 \AA$. This spacing of magnetic moments is just slightly larger than that of $\operatorname{GdN}(3.51 \AA)$. Since hcp and fcc structures have exactly the same number of the first and second $\mathrm{NN}$ atoms, it is not surprising that, similar to $\mathrm{Gd}$ metal, the $J_{1}$ exchange in GdN is ferromagnetic; just the value of $J_{1}=0.86 \mathrm{meV}$ in GdN is much smaller than $J_{1} \approx 7.0 \mathrm{meV}$ in Gd metal, due to GdN's poor metallicity. The superexchange contributions in GdN are not strong enough to offset those contributions from the RKKY-type ferromagnetic interactions. Thus GdN is ferromagnetic with a low transition temperature $(58 \mathrm{~K})$. With the increase of anion size, the superexchange is strengthened causing the $J_{2}$ parameter to decrease. In addition, the $J_{1}$ exchange also becomes antiferromagnetic. The variation of $J_{1}$ as a function of the lattice strain indicates that this variation is caused by the RKKY-type indirect exchange interaction. However, we do not exclude the possibility of the contribution from the $90^{\circ}$-superexchange interaction that can be quite large in some oxides. ${ }^{26}$ Generally speaking the overall effect, after the competition between the various magnetic interactions is taken into account, is that antiferromagnetic ordering gains the upper hand, though the Néel temperature is rather small, but the antiferromagnetic ordering increases with the pnictide anion size.

Based on the predicted interplay between the exchange coupling, the pnictide anion size, and the lattice constant, we suggest that there likely exist gadolinium compounds in which both $J_{1}$ and $J_{2}$ are ferromagnetic. Such materials might have larger effective exchange coupling and higher transition temperature than those of the simple stoichiometric rare-earth compounds. An example of this is the higher $T_{C}$ obtained by doping GdN with carbon. ${ }^{27}$

In summary, we find that there are profound changes in the magnetic ordering behavior between pure $\mathrm{Gd}, \mathrm{GdN}$, and GdP. Density functional theory with the Hubbard correlation terms gives an adequate description of the magnetic properties of Gd monopnictides. We have been able to deduce the exchange parameters of these systems directly from ab initio calculations and to verify them by MC simulations. The magnetic orderings of Gd monopnictides are determined by the interplay between the first and second NN exchange interactions. Our results clearly demonstrate the coexistence of the RKKY-type magnetic interaction and the superexchange interaction, the relative contribution of which changes with the ionic radius of pnictides.

The authors acknowledge invaluably helpful discussions with P. W. Anderson, A. G. Petukhov, W. Nolting, and A. Sharma. This work was supported by NSF (Grant Nos. DMR-0203359, MRSEC-DMR-0213808, and EPS-9720643) and the Nebraska Research Initiative.

${ }^{1}$ H. R. Child, M. K. Wilkinson, J. W. Cable, W. C. Koehler, and E. O. Wollan, Phys. Rev. 131, 922 (1963).

${ }^{2}$ G. T. Trammell, Phys. Rev. 131, 932 (1963).

${ }^{3}$ A. Hasegawa and A. Yanase, J. Phys. Soc. Jpn. 42, 492 (1977).

${ }^{4}$ R. Monnier, J. Rhyner, T. M. Rice, and D. D. Koelling, Phys. Rev. B 31, 5554 (1985).

${ }^{5}$ G. Travaglini, F. Marabelli, R. Monnier, E. Kaldis, and P. Wachter, Phys. Rev. B 34, 3876 (1986).

${ }^{6}$ J.-B. Xia, S.-F. Ren, and Y.-C. Chang, Phys. Rev. B 43, 1692 (1991).

7 A. I. Liechtenstein, V. P. Antropov, and B. N. Harmon, Phys. Rev. B 49, 10770 (1994).

${ }^{8}$ A. G. Petukhov, W. R. L. Lambrecht, and B. Segall, Phys. Rev. B 53, 3646 (1996).

${ }^{9}$ W. R. L. Lambrecht, B. Segall, A. G. Petukhov, R. Bogaerts, and F. Herlach, Phys. Rev. B 55, 9239 (1997).

${ }^{10}$ C. Stampfl, W. Mannstradt, R. Asahi, and A. J. Freeman, Phys. Rev. B 63, 155106 (2001).

${ }^{11}$ Chun-Gang Duan, R. F. Sabiryanov, Jianjun Liu, W. N. Mei, P. A. Dowben, and J. R. Hardy, J. Appl. Phys. 97, 10A915 (2005).

${ }^{12}$ S. J. Allen, Jr., N. Tabatabaie, C. J. Palmstrøm, G. W. Hull, T. Sands, F. DeRosa, H. L. Gilchrist, and K. C. Garrison, Phys. Rev. Lett. 62, 2309 (1989).

${ }^{13}$ T. Chattopadhyay, P. Burlet, J. Rossat-Mignod, H. Bartholin, C. Vettier, and O. Vogt, Phys. Rev. B 49, 15096 (1994).

${ }^{14}$ D. X. Li, Y. Haga, H. Shida, T. Suzuki, Y. S. Kwon, and G. Kido, J. Phys.: Condens. Matter 9, 10777 (1997).

${ }^{15}$ For a complete review, see the series Handbook on Physics and Chemistry of Rare Earth (North-Holland, Amsterdam, 1979).

${ }^{16}$ D. E. Brehmer, K. Zhang, C. J. Schwarz, S. P. Chau, and S. J. Allen, Appl. Phys. Lett. 67, 1268 (1995).

${ }^{17}$ Ph. Kurz, G. Bihlmayer, and S. Blügel, J. Phys.: Condens. Matter 14, 6353 (2002)

${ }^{18}$ P. W. Anderson, Phys. Rev. 79, 350 (1950); 115, 2 (1959).

${ }^{19}$ Chun-Gang Duan, R. F. Sabiryanov, Jianjun Liu, W. N. Mei, P. A. Dowben, and J. R. Hardy, Phys. Rev. Lett. 94, 237201 (2005).

${ }^{20}$ J. Samuel Smart, Effective Field Theories of Magnetism (Saunders, Philadelphia, 1966), pp. 76-77.

${ }^{21}$ K. Schwarz, P. Blaha, and G. K. H. Madsen, Comput. Phys. Commun. 147, 71 (2002).

${ }^{22}$ V. I. Anisimov, I. V. Solovyev, M. A. Korotin, M. T. Czyzyk, and G. A. Sawatzky, Phys. Rev. B 48, 16929 (1993).

${ }^{23}$ V. A. Gubanov, A. V. Postnikov, and A. I. Likhtenshtein, Magnetism and the Electronic Structure of Crystals (Springer, New York, 1992), p. 23.

${ }^{24}$ W. A. Harrison, Electronic Structure and The Properties of Solids (Freeman, San Francisco, 1980), pp. 451, 481, and 505.

${ }^{25}$ H. E. Nigh, S. Legvold, and F. H. Spedding, Phys. Rev. 132, 1092 (1963).

${ }^{26}$ W. Geertsma and D. Khomskii, Phys. Rev. B 54, 3011 (1996).

${ }^{27}$ M. Kuznietz, J. Appl. Phys. 42, 1470 (1971). 